\title{
A NEW GENERALIZED VARENTROPY AND ITS PROPERTIES
}

\author{
S. Maadani ${ }^{1}$, G. R. Mohtashami Borzadaran ${ }^{2}$, A. H. Rezaei Roknabadi ${ }^{3}$ \\ Ferdowsi University of Mashhad, Azadi Square, Mashhad, Iran \\ ${ }^{1}$ madani.sa@mail.um.ac.ir, $\quad{ }^{2}$ grmohtashami@um.ac.ir, $\quad{ }^{3}$ rezaei@um.ac.ir
}

\begin{abstract}
The variance of Shannon information related to the random variable $X$, which is called varentropy, is a measurement that indicates, how the information content of $X$ is scattered around its entropy and explains its various applications in information theory, computer sciences, and statistics. In this paper, we introduce a new generalized varentropy based on the Tsallis entropy and also obtain some results and bounds for it. We compare the varentropy with the Tsallis varentropy. Moreover, we explain the Tsallis varentropy of the order statistics and analyse this concept in residual (past) lifetime distributions and then introduce two new classes of distributions by them.
\end{abstract}

Keywords: Generalized varentropy, Past Tsallis varentropy, Residual Tsallis varentropy, Tsallis varentropy, Varentropy.

\section{Introduction}

Nowadays, the use of information measures has an essential role in analyzing statistical issues and is greatly considered by the statisticians. Shannon [21] introduced a measure of uncertainty for the discrete random variable $X$ with probability mass function $P(x)$ to form into $E(-\log P(X))$, which is a basis for the information theory. The generalization of Shannon's measure for continuous random variable $X$ with density function $f(x)$ and support $S$, which is named a differential entropy, reads as follows:

$$
h(X)=-\int_{S} f(x) \log f(x) d x .
$$

This measure is the expectation of random variable $(-\log f(X))$ and has recently attracted the attention of researchers.

In computer sciences, the variance of $(-\log p(X))$ of the discrete random variable $X$ is called the varentropy. This measure is an essential factor of the optimal code length calculation in the data compression process, dispersion of sources, and so on. To conduct further studies, we refer the reader to $[3,7,15]$. Since the varentropy was defined for discrete random variables, in this paper, we focus on the varentropy for continuous random variables, and we discuss it under the same name.

Let $X$ be a continuous random variable with density function $f$. Then the varentropy of $X$ is defined as

$$
V E(X)=\operatorname{Var}(-\log f(X))=E[-\log f(X)-h(X)]^{2},
$$

where $V E(X)$ is called the varentropy of $X$. Unfortunately, there are not many studies on the varentropy in the field of statistics. Song [22] introduced $V E$ (of course not with that name), as an intrinsic measure of distributions shape, which can be an excellent alternative for the kurtosis measure. When the traditional kurtosis measure is not measurable, as Student's t distributions with degrees of freedom less than four, Cauchy and Pareto distributions, $V E$ is a measure that can be used to compare the heavy-tailed distributions instead of kurtosis measure. 
Liu [16] studied $V E$ under the concept of information volatility and introduced some mathematical properties of it. He calculated $V E$ for some distributions and showed that $V E$ of gamma, beta (with parameters $(\alpha, \alpha)$ when $\alpha<2-\sqrt{2}$ ) and normal distributions are more than less than, and equal to $1 / 2$ respectively, and that $V E$ of the uniform distributions is zero. Therefore $V E$ can separate the gamma, normal, beta and uniform distributions. He showed that $V E$ of the generalized Gaussian distribution is exactly the reciprocal of its shape parameter, which gives us a new method to estimate this parameter. Zografos [29] found an empirical estimator for Song's measure in the elliptic multivariate distributions. Enomoto et al. [13] considered the multivariate normality test based on the sample measure of multivariate kurtosis defined by Song [22]. Afhami et al. [2] introduced the goodness of fit test based on entropy and varentropy of $k$-record values for the generalized Pareto distribution and more recently, in addition to the above, the application of the varentropy in reliability theory has been conducted in [10].

A generalization of the Shannon entropy is the Tsallis entropy (see [23]). Let $X$ be a continuous random variable with density function $f$. Then the Tsallis entropy of order $\alpha$ for $X$ is defined as

$$
I_{T}(X, \alpha)=\frac{1}{(1-\alpha)}\left(\int_{s} f^{\alpha}(x) d x-1\right), \quad \alpha>0, \quad \alpha \neq 1,
$$

and if $\alpha \rightarrow 1$, then the Tsallis entropy is reduced to (1.1). The Tsallis entropy has many applications in physics, statistical mechanics and image processing. The properties of the Tsallis entropy have been investigated by several authors, see papers [17, 24, 25, 28].

On the other hand, the concentration of measure principle is one of the cornerstones in geometric functional analysis and probability theory, and it is widely used in many other areas. Hence the concentration property of information content $(-\log f(X))$ is one of the central interests in information theory, and it has great relevance with various other areas such as probability theory, and the varentropy is the measure of this concentration. Suppose that $X$ and $Y$ are two random variables with the same Shannon entropy; for example, the Shannon entropy is zero in both standard uniform and the exponential (with the parameter $e$ ) distributions. Can we say that the uncertainty criterion is the same in both random variables? In our opinion, our confidence in the measured value depends on the degree of information dispersion around the entropy. Therefore, for random variables with the less varentropy the uncertainty criteria are more appropriate. This concept is valid for the measure of the Tsallis uncertainty information, and if two random variables have the same Tsallis entropy, the Tsallis varentropy indicates which of these random variables has the more appropriate criterion for Tsallis uncertainty.

The purpose of this paper is to generalize Shannon's varentropy based on the Tsallis entropy, and compare its properties with Shannon's varentropy and extend it in the field of order statistics and reliability theory.

This paper contains the following sections. The generalized varentropy which we call $T V E$ is introduced in Section 2. We also obtain some of its properties and compare $T V E$ with $V E$ in this section. In Section 3 we discuss the Tsallis varentropy of the order statistics. In Section 4, we study $T V E$ in lifetime researches and achieve some bounds for it by hazard rate and reversed hazard rate functions. Moreover, we examine the effects of system's age on TVE. Finally, in Section 5, we introduce two new classes of distributions by residual and past Tsallis varentropy.

\section{Introduction of Tsallis Varentropy}

Let $X$ be a continuous random variable with density function $f$. Then Tsallis entropy of order $\alpha$ for $X$ is the expectation of a random variable $\left(f^{\alpha}(X)-1\right) /(1-\alpha)$ and $T V E$ is the variance of it. Following what was said above, we define $T V E$ and introduce some properties of this measure. 
Definition 1. For the continuous random variable $X$ with density function $f$, the Tsallis varentropy of order $\alpha$ for $X$ is defined as follows:

$$
\begin{aligned}
\operatorname{TVE}(X, \alpha) & =\frac{1}{(1-\alpha)^{2}} \operatorname{Var}\left(f^{\alpha-1}(X)\right) \quad \alpha>0 \quad \alpha \neq 1 \\
& =\frac{1}{(1-\alpha)^{2}}\left(\int f^{2 \alpha-1}(x) d x-\left(\int f^{\alpha}(x) d x\right)^{2}\right),
\end{aligned}
$$

where $\operatorname{TVE}(X, \alpha)$ is the Tsallis varentropy of order $\alpha$ for $X$. It is clear that when $\alpha \rightarrow 1,(2.1)$ implies (1.2).

For example, if $X \sim \operatorname{Exp}(\theta)$ with density function $f(x)=\theta e^{-\theta x}(x>0, \theta>0)$, then

$$
\operatorname{TEV}(X, \alpha)=\frac{1}{(1-\alpha)^{2}}\left(\frac{\theta^{2 \alpha-2}}{2 \alpha-1}-\left(\frac{\theta^{\alpha-1}}{\alpha}\right)^{2}\right)=\frac{\theta^{2 \alpha-2}}{\alpha^{2}(2 \alpha-1)}, \quad \alpha>\frac{1}{2} .
$$

We see that $\lim _{\alpha \rightarrow 1} \operatorname{TVE}(X, \alpha)=1$ and that $\operatorname{TVE}(X, 1)=1$ is the Shannon varentropy of the exponential distribution.

Remark 1. If $X \sim \operatorname{Exp}(\theta)$ and $0<\alpha \leq 1 / 2$, then $\operatorname{TVE}(X, \alpha)$ diverges to infinity.

Theorem 1. $X$ has a uniform distribution if and only if $T V E(X, \alpha)=0$ for all $\alpha>0$.

P r o o f. If $X \sim U(a, b)$ with density function $f(x)=1 /(b-a) \quad a<x<b$, then

$$
\operatorname{TEV}(X, \alpha)=\frac{1}{(1-\alpha)^{2}}\left[(b-a)^{2-2 \alpha}-\left((b-a)^{1-\alpha}\right)^{2}\right]=0 .
$$

On the other hand, if $\operatorname{TVE}(X, \alpha)=0$, then $\operatorname{Var}\left(f^{\alpha-1}(X)\right)=0$, so $f(X)$ is almost surely constant. Suppose that $f(X)=c$ (if $a<X<b$ ) is the support of $X$, then

$$
\int_{a}^{b} f(x) d x=\int_{a}^{b} c d x \quad \text { and } \quad c=\frac{1}{b-a}
$$

Liu [16] showed that if $X$ is a continuous random variable with symmetric density function $f$ with respect to $x=a$, then $V E(|X|)=V E(X)$.

Proposition 1. Suppose that $X$ is a continuous random variable with a symmetric density function $f$ with respect to $x=a$. Then

$$
T V E(|X|, \alpha)=2^{2 \alpha-2} T V E(X, \alpha) .
$$

P r o o f. Without loss of generality suppose $a=0$. In this case the density function $g(x)$ of the random variable $|X|$ is $g(x)=f(-x)+f(x)=2 f(x)$, and hence

$$
\begin{aligned}
& T V E(|X|, \alpha)=\frac{1}{(1-\alpha)^{2}}\left(\int_{0}^{\infty}(2 f(x))^{2 \alpha-1} d x-\left[\int_{0}^{\infty}(2 f(x))^{\alpha} d x\right]^{2}\right) \\
& =\frac{2^{2 \alpha-2}}{(1-\alpha)^{2}}\left(\int_{-\infty}^{\infty} f^{2 \alpha-1}(x) d x-\left[\int_{-\infty}^{\infty} f^{\alpha}(x) d x\right]^{2}\right)=2^{2 \alpha-2} T V E(X, \alpha) .
\end{aligned}
$$


For example, if $X$ has the Laplace distribution with density function $f(x)=\frac{1}{2 \beta} e^{-1 / \beta \cdot|x|}$, then we can show that

$$
T V E(X, \alpha)=\frac{(2 \beta)^{2-2 \alpha}}{\alpha^{2}(2 \alpha-1)}, \quad \alpha>\frac{1}{2} .
$$

On the other hand if $X \sim \operatorname{Laplace}(0, \beta)$, then $|X| \sim \operatorname{Exp}(1 / \beta)$. Therefore by using (2.2), we have

$$
T V E(|X|, \alpha)=\frac{(1 / \beta)^{2 \alpha-2}}{\alpha^{2}(2 \alpha-1)}, \quad \alpha>\frac{1}{2} .
$$

It implies that $T V E(|X|, \alpha)=2^{2 \alpha-2} T V E(X, \alpha)$. It is obvious that if $\alpha \rightarrow 1$, then $V E(|X|)=$ $V E(X)$.

One of the most important properties of $V E$ is the following:

The varentropy is a scale and location invariant measure so $V E(a X+b)=V E(X)$ for all $a, b \in \mathbb{R}$. This property implies that in the location and scale family of distributions, $V E$ is independent of the distribution parameters. Therefore the empirical estimation of $V E$ can separate the distribution of this family. Now the question arises, is $T V E$ an affine invariant measure? To answer this question, let us look at the following theorem and at the next example.

Theorem 2. Suppose that $X$ is a continuous random variable and that $f(x)$ is its density function. Then

$$
T V E(a X+b, \alpha)=a^{2-2 \alpha} T V E(X, \alpha) .
$$

P r o o f. If $Y=g(X)$ and $g(X)$ is a strictly monotone function of $X$, then

$$
f_{Y}(y)=\frac{f\left(g^{-1}(y)\right)}{g^{\prime}\left(g^{-1}(y)\right)}
$$

It is easy to see that

$$
T V E(g(X), \alpha)=\frac{1}{(1-\alpha)^{2}} \operatorname{Var}\left(\left(\frac{f(X)}{g^{\prime}(X)}\right)^{\alpha-1}\right) .
$$

Therefore if $g(X)=a X+b$, then $T V E(a X+b, \alpha)=a^{2-2 \alpha} T V E(X, \alpha)$.

Theorem 2 implies that in the location and scale family of distributions, the Tsallis varentropy is independent of the location parameter but it depends on the scale parameter.

For example, if $X \sim N\left(\mu, \sigma^{2}\right)$, then $T V E$ of $X$ is

$$
T E V(X, \alpha)=\left(2 \pi \sigma^{2}\right)^{1-\alpha} \times \frac{1 / \sqrt{2 \alpha-1}-1 / \alpha}{(1-\alpha)^{2}}, \quad \alpha>\frac{1}{2} .
$$

We can see that if $\alpha \rightarrow 1, T V E(X, 1)=V E(X)=1 / 2$, and $T V E$ is reduced to $V E$ of normal distribution, then we can see that $T V E$ is dependent on the scale parameter $\sigma^{2}$.

Definition 2. The Tsallis varentropy of order $\alpha$ for a random vector $\mathbf{X}=\left(X_{1}, X_{2}, \ldots ., X_{n}\right)$ with joint density function $f(\mathbf{x})$, is defined as follows:

$$
T V E(\mathbf{X}, \alpha)=\frac{1}{(1-\alpha)^{2}}\left(\int_{\mathbb{R}^{n}} f^{2 \alpha-1}(\mathbf{x}) d \mathbf{x}-\left(\int_{\mathbb{R}^{n}} f^{\alpha}(\mathbf{x}) d \mathbf{x}\right)^{2}\right), \quad \alpha>0, \quad \alpha \neq 1 .
$$

Theorem 3. If $\mathbf{X}$ is an $n$-dimensional random variable, then for any invertible $n \times n$ matrix $A$ and any $n \times 1$ vector $B$ we have $\operatorname{TVE}(A \mathbf{X}+B, \alpha)=|A|^{2-2 \alpha} \operatorname{TVE}(\mathbf{X}, \alpha)$, where $|A|$ is the determinant of the matrix A. 
Table 1. Comparison $V E(X)$ and $T V E(X, \alpha)$ (here $\dot{\psi}(\cdot), \Gamma(\cdot)$ and $B(a, b)$ are trigamma, gamma and beta functions respectively).

\begin{tabular}{|c|c|c|c|}
\hline Distribution & Density function & $V E(X)$ & $T V E(X, \alpha)$ \\
\hline uniform $(a, b)$ & $f(x)=\frac{1}{b-a}$ & 0 & 0 \\
\hline exponential & $\begin{array}{l}f(x)=\theta e^{-\theta x} \\
\theta>0, x>0\end{array}$ & 1 & $\frac{\theta^{2 \alpha-2}}{\alpha^{2}(2 \alpha-1)}, \quad \alpha>\frac{1}{2}$ \\
\hline Laplace & $\begin{array}{c}f(x)=e^{-|x-\mu| / \sigma} / 2 \sigma \\
\sigma>0\end{array}$ & 1 & $\frac{(2 \sigma)^{2-2 \alpha}}{\alpha^{2}(2 \alpha-1)}, \quad \alpha>\frac{1}{2}$ \\
\hline Pareto & $\begin{array}{c}f(x)=\theta \beta^{\theta} / x^{\theta+1} \\
\beta>0, \theta>0, x>\beta\end{array}$ & $(\theta+1)^{2} \times \frac{1}{\theta^{2}}$ & $\frac{\beta^{2-2 \alpha} \theta^{2 \alpha}}{(1-\alpha)^{2}}\left\{\frac{1}{\theta(\theta+1)(2 \alpha-1)-\theta}-\frac{1}{[\alpha(\theta+1)-1]^{2}}\right\}$ \\
\hline normal & $f(x)=\frac{e^{-(x-\mu)^{2} /\left(2 \sigma^{2}\right)}}{\sqrt{2 \pi \sigma^{2}}}$ & $\frac{1}{2}$ & $(1-\alpha)^{-2}\left(2 \pi \sigma^{2}\right)^{1-\alpha}\left(\frac{1}{\sqrt{2 \alpha-1}}-\frac{1}{\alpha}\right), \quad \alpha>\frac{1}{2}$ \\
\hline gamma & $\begin{array}{c}f(x)=\frac{\lambda^{\theta}}{\Gamma(\theta)} x^{\theta-1} e^{-\lambda x} \\
\theta>0, \lambda>0, x>0\end{array}$ & $(\theta-1)^{2} \dot{\psi}(\theta)-\theta+2$ & $\begin{array}{c}(1-\alpha)^{-2}\left(\frac{\lambda^{\theta}}{\Gamma(\theta)}\right)^{2 \alpha-1}\left\{\frac{\Gamma((2 \alpha-1)(\theta-1)+1)}{[(2 \alpha-1) \lambda]^{(2 \alpha-1)(\theta-1)+1}}\right. \\
\left.-\frac{\lambda^{\theta}[\Gamma(\alpha(\theta-1)+1)]^{2}}{\Gamma(\theta)(\alpha \lambda)^{2 \alpha(\theta-1)+2}}\right\}, \quad \alpha>\frac{1}{2}\end{array}$ \\
\hline Weibull & $\begin{array}{c}f(x)=\theta \lambda^{\theta} x^{\theta-1} e^{-(\lambda x)^{\theta}} \\
\theta>0, \lambda>0, x>0\end{array}$ & $\dot{\psi}(1)\left(1-\theta^{-1}\right)^{2}+2 \theta^{-1}-1$ & $\begin{array}{c}\frac{(\theta \lambda)^{2 \alpha-2}}{(1-\alpha)^{2}}\left\{\frac{\Gamma\left(\theta^{-1}(2 \alpha-1)(\theta-1)+\theta^{-1}\right)}{(2 \alpha-1)^{\left[(2 \alpha-1)(\theta-1) \theta^{-1}+\theta^{-1}\right]}}\right. \\
\left.-\frac{\Gamma^{2}\left(\theta^{-1} \alpha(\theta-1)+\theta^{-1}\right)}{\alpha^{\left[2 \theta^{-1} \alpha(\theta-1)+2 \theta^{-1}\right]}}\right\}\end{array}$ \\
\hline beta & $\begin{array}{c}f(x)=\frac{x^{m-1}(1-x)^{n-1}}{B(m, n)} \\
0<x<1, m>0, n>0\end{array}$ & $(m-1)^{2} \dot{\psi}(m)+(n-1)^{2} \dot{\psi}(n)$ & $\begin{array}{c}\frac{B(m, n)^{1-2 \alpha}}{(1-\alpha)^{2}}\{B((2 \alpha-1)(m-1)+1,(2 \alpha-1)(n-1)+1) \\
\left.-B^{-1}(m, n) B^{2}(\alpha(m-1)+1, \alpha(n-1)+1)\right\}\end{array}$ \\
\hline Rayleigh & $\begin{aligned} f(x) & =\frac{x}{\sigma^{2}} e^{-x^{2} /\left(2 \sigma^{2}\right)} \\
x & >0, \sigma>0\end{aligned}$ & $\frac{1}{4} \dot{\psi}(1)$ & $\frac{2^{\alpha-1} \sigma^{2-2 \alpha}}{(1-\alpha)^{2}}\left\{\frac{\Gamma(\alpha)}{(2 \alpha-1)^{\alpha}}-\frac{\Gamma^{2}((\alpha+1) / 2)}{\alpha^{\alpha+1}}\right\}$ \\
\hline
\end{tabular}

P r o o f. The proof is similar to Theorem 2 in the $n$-dimensional spaces.

Remark 2. Theorems 2 and 3 indicate that $T V E$ is a location-invariant measure but is not the scale-invariant, unless $\alpha \rightarrow 1$.

Remark 3. If $X$ and $Y$ are two random variables, $X \sim \operatorname{Exp}(\theta), Y \sim N\left(\mu, \sigma^{2}\right)$ and $\operatorname{Var}(X)=$ $\operatorname{Var}(Y)$ then

$$
T V E(X, \alpha)=k(\alpha) T V E(Y, \alpha), \quad \alpha>\frac{1}{2}
$$

where

$$
k(\alpha)=(2 \pi)^{\alpha-1} \frac{\alpha+\sqrt{2 \alpha-1}}{\alpha \sqrt{2 \alpha-1}},
$$

and if $\alpha \rightarrow 1$, then $V E(X)=2 V E(Y)$.

In Table 1, we compare the $V E$ and $T V E$ for some continuous distributions.

Theorem 4. Let $X_{1}, X_{2}, \ldots X_{n}$ be independent random variables with joint density function $f(\mathbf{x})$. Then 


$$
\begin{aligned}
\operatorname{TVE}\left(X_{1}, X_{2}, \ldots, X_{n}, \alpha\right) & =\frac{1}{(1-\alpha)^{2}} \prod_{i=1}^{n}\left\{(1-\alpha)^{2} \operatorname{TVE}\left(X_{i}, \alpha\right)+\left[(1-\alpha) I_{T}\left(X_{i}, \alpha\right)+1\right]^{2}\right\} \\
& -\frac{1}{(1-\alpha)^{2}} \prod_{i=1}^{n}\left\{\left[(1-\alpha) I_{T}\left(X_{i}, \alpha\right)+1\right]^{2}\right\}
\end{aligned}
$$

and when $\alpha \rightarrow 1$, (2.3) reduces to

$$
T V E\left(X_{1}, X_{2}, \ldots, X_{n}, 1\right)=V E\left(X_{1}, X_{2}, \ldots, X_{n}\right)=\sum_{i=1}^{n} V E\left(X_{i}\right) .
$$

P r o o f. If $X_{1}, X_{2}, \ldots, X_{n}$ are independent random variables, we know that

$$
\operatorname{Var}\left(\prod_{i=1}^{n} X_{i}\right)=\prod_{i=1}^{n}\left[\operatorname{Var}\left(X_{i}\right)+E^{2}\left(X_{i}\right)\right]-\prod_{i=1}^{n} E^{2}\left(X_{i}\right) .
$$

Since $f\left(x_{1}\right), \ldots, f\left(x_{n}\right)$ are marginal density functions of $f(\mathbf{x})$ and $f^{\alpha-1}\left(X_{1}\right), \ldots, f^{\alpha-1}\left(X_{n}\right)$ are independent random variables, (2.4) implies that

$$
\begin{gathered}
\operatorname{TVE}\left(X_{1}, X_{2}, . . X_{n}, \alpha\right)=\frac{1}{(1-\alpha)^{2}} \operatorname{Var}\left(\prod_{i=1}^{n} f^{\alpha-1}\left(X_{i}\right)\right) \\
=\frac{1}{(1-\alpha)^{2}} \prod_{i=1}^{n} \operatorname{Var}\left(f^{\alpha-1}\left(X_{i}\right)+E^{2}\left(f^{\alpha-1}\left(X_{i}\right)\right)\right)-\frac{1}{(1-\alpha)^{2}} \prod_{i=1}^{n} E^{2}\left(f^{\alpha-1}\left(X_{i}\right)\right) .
\end{gathered}
$$

Equation (1.3) indicates that $E\left(f^{\alpha-1}(X)\right)=(1-\alpha) I_{T}(X, \alpha)+1$, and (2.1) implies

$$
\operatorname{Var}\left(f^{\alpha-1}(X)\right)=(1-\alpha)^{2} T V E(X, \alpha) .
$$

Therefore

$$
\begin{aligned}
T V E\left(X_{1}, X_{2}, \ldots, X_{n}, \alpha\right) & =\frac{1}{(1-\alpha)^{2}} \prod_{i=1}^{n}\left\{(1-\alpha)^{2} \operatorname{TVE}\left(X_{i}, \alpha\right)+\left[(1-\alpha) I_{T}\left(X_{i}, \alpha\right)+1\right]^{2}\right\} \\
& -\frac{1}{(1-\alpha)^{2}} \prod_{i=1}^{n}\left\{\left[(1-\alpha) I_{T}\left(X_{i}, \alpha\right)+1\right]^{2}\right\} .
\end{aligned}
$$

It is obvious that when $\alpha \rightarrow 1$, by using L'hopital's rule, we have

$$
T V E\left(X_{1}, X_{2}, \ldots, X_{n}, 1\right)=V E\left(X_{1}, X_{2}, \ldots, X_{n}\right)=\sum_{i=1}^{n} V E\left(X_{i}\right) .
$$

Corollary 1. If $X$ and $Y$ are two independent random variables with joint density function $f(x, y)$ and marginal density functions $f_{X}(x)$ and $f_{Y}(y)$, respectively, then

$$
\begin{gathered}
T V E((X, Y), \alpha)=(1-\alpha)^{2} \operatorname{TVE}(X, \alpha) T V E(Y, \alpha)+T V E(X, \alpha)\left[(1-\alpha) I_{T}(Y, \alpha)+1\right]^{2} \\
+T V E(Y, \alpha)\left[(1-\alpha) I_{T}(X, \alpha)+1\right]^{2},
\end{gathered}
$$

where $I_{T}(X, \alpha)$ and $I_{T}(Y, \alpha)$ are Tsallis entropies of $X$ and $Y$ respectively, and (2.5) implies that $T V E((X, Y), 1)=V E(X, Y)=V E(X)+V E(Y)$. 
Corollary 2. By using (2.5), the following inequalities are valid:

(a) $\operatorname{TVE}((X, Y), \alpha)>(1-\alpha)^{2} T V E(X, \alpha) T V E(Y, \alpha)$.

(b) $T V E((X, Y), \alpha)>T V E(X, \alpha)\left[(1-\alpha) I_{T}(Y, \alpha)+1\right]^{2}+T V E(Y, \alpha)\left[(1-\alpha) I_{T}(X, \alpha)+1\right]^{2}$.

Corollary 3. If $X_{1}, X_{2}, \ldots, X_{n}$ are iid random variables, then using Theorem 4 we have

$$
\begin{aligned}
T V E\left(X_{1}, X_{2}, \ldots, X_{n}, \alpha\right)= & \frac{1}{(1-\alpha)^{2}}\left\{(1-\alpha)^{2} T V E\left(X_{1}, \alpha\right)+\left[(1-\alpha) I_{T}\left(X_{1}, \alpha\right)+1\right]^{2}\right\}^{n} \\
& -\frac{1}{(1-\alpha)^{2}}\left\{\left[(1-\alpha) I_{T}\left(X_{1}, \alpha\right)+1\right]^{2}\right\}^{n} .
\end{aligned}
$$

Theorem 5. Let $X$ and $Y$ be two random variables with joint density function $f(x, y)$ and conditional density function $f(x \mid y)$. If

$$
E\left(f^{2 \alpha-2}(X \mid Y)\right) \cdot E\left(f^{2 \alpha-2}(Y)\right) \geq\left[E\left(f^{\alpha-1}(X, Y)\right)\right]^{2},
$$

then

$$
T V E((X, Y), \alpha) \geq(1-\alpha)^{-2} \operatorname{Cov}\left(f^{2 \alpha-2}(X \mid Y), f^{2 \alpha-2}(Y)\right),
$$

and the equality established when $X$ and $Y$ are independent.

P r o o f. The joint density of $X$ and $Y$ is $f(x, y)=f(x \mid y) \cdot f(y)$ therefore,

$$
\begin{gathered}
\operatorname{TVE}((X, Y), \alpha)=\frac{1}{(1-\alpha)^{2}} \operatorname{Var}\left(f^{\alpha-1}(X \mid Y) \cdot f^{\alpha-1}(Y)\right) \\
=\frac{1}{(1-\alpha)^{2}}\left\{E\left(f^{2 \alpha-2}(X \mid Y) \cdot f^{2 \alpha-2}(Y)\right)-\left[E\left(f^{\alpha-1}(X \mid Y) \cdot f^{\alpha-1}(Y)\right)\right]^{2}\right\} .
\end{gathered}
$$

Using covariance definition we have

$$
\operatorname{Cov}\left(f^{2 \alpha-2}(X \mid Y), f^{2 \alpha-2}(Y)\right)=E\left(f^{2 \alpha-2}(X \mid Y), f^{2 \alpha-2}(Y)\right)-E\left(f^{2 \alpha-2}(X \mid Y)\right) \cdot E\left(f^{2 \alpha-2}(Y)\right),
$$

therefore,

$$
\begin{gathered}
T V E((X, Y), \alpha)=\frac{1}{(1-\alpha)^{2}}\left\{\operatorname{Cov}\left(f^{2 \alpha-2}(X \mid Y), f^{2 \alpha-2}(Y)\right)+E\left(f^{2 \alpha-2}(X \mid Y)\right) \cdot E\left(f^{2 \alpha-2}(Y)\right)\right. \\
\left.-\left[E\left(f^{\alpha-1}(X, Y)\right)\right]^{2}\right\}
\end{gathered}
$$

If (2.6) holds, then (2.7) will be easily obtained.

\section{Tsallis varentropy of order $\alpha$ for order statistics}

Suppose that $X_{1}, X_{2}, \ldots, X_{n}$ are independent and identically distributed observations from density and cumulative function $f$ and $F$, respectively. If we arrange of $X_{1}, X_{2}, \ldots, X_{n}$ from the smallest to the largest denoted as $X_{1: n} \leq X_{2: n} \leq \cdots \leq X_{n: n}$ and $f_{i: n}$ denotes the density function of the $i$ th order statistic, then

$$
f_{i: n}(x)=\frac{1}{B(i, n-i+1)}[F(x)]^{i-1}[1-F(x)]^{n-i} f(x),
$$


where

$$
B(a, b)=\int_{0}^{1} x^{a-1}(1-x)^{b-1} d x, \quad a>0, \quad b>0 .
$$

The order statistics have many applications in probability and statistics, as the characterization of distributions, goodness-of-fit test, reliability engineering, and many other problems. For more information, we refer the reader to $[4,8]$. The order statistics also have been studied widely in information theory in $[5,12,18,26,27]$. Furthermore, the stochastic order is also has many applications in finance, risk theory, management science and biomathematics. For example, we refer the reader to scholarly researches such as $[1,6,9,11,14,19,20]$. In this section, we introduce the Tsallis varentropy of order $\alpha$ for the $i$ th order statistic. This measure can be one of the useful information measures for system designers. We know that one of the systems in reliability engineering is an $(n-i+1)$-out-of- $n$ system, and the system is active, when at least $(n-i+1)$ components are operating. Assume that $X_{1}, X_{2}, \ldots, X_{n}$ denote the identical lifetime of the system components. Then the $i$ th order statistic indicates the lifetime of the systems. In special cases, $X_{1: n}$ and $X_{n: n}$ are the lifetime of the series and parallel systems, respectively. Therefore the Tsallis entropy of the $i$ th order statistic is a measure of the uncertainty of the lifetime system and the Tsallis varentropy is the volatility of this information.

Definition 3. Let $X_{1}, X_{2}, \ldots, X_{n}$ be a random sample from a continuous distribution with density function $f$. Let $X_{i: n}$ denotes the ith order statistic. The Tsallis varentropy of $i$ th order statistics is defined as:

$$
T V E\left(X_{i: n}, \alpha\right)=\frac{1}{(1-\alpha)^{2}} \operatorname{Var}\left(f^{\alpha-1}\left(X_{i: n}\right)\right)=(1-\alpha)^{-2}\left\{\int_{S} f_{i: n}^{2 \alpha-1}(x) d x-\left(\int_{S} f_{i: n}^{\alpha}(x) d x\right)^{2}\right\},
$$

where $S$ is the support of $X_{i: n}$.

In the following theorem we introduce a method for calculating the Tsallis varentropy for $i$ th order statistic.

Theorem 6. Suppose that $X$ is a continuous random variable with density function $f$ and cumulative distribution function $F$, and let $X_{i: n}$ denote the $i$ th order statistic. Then the Tsallis varentropy of $X_{i: n}$ can be expressed as:

$$
T V E\left(X_{i: n}, \alpha\right)=(1-\alpha)^{-2}\left[A_{i: n}(\alpha)-\left(B_{i: n}(\alpha)\right)^{2}\right],
$$

where

$$
A_{i: n}(\alpha)=\frac{B((2 \alpha-1)(i-1)+1,(2 \alpha-1)(n-i)+1)}{B^{2 \alpha-1}(i, n-i+1)} E\left(f^{2 \alpha-2}\left(F^{-1}\left(T_{i}\right)\right)\right),
$$

and

$$
B_{i: n}(\alpha)=\frac{B(\alpha(i-1)+1, \alpha(n-i)+1)}{B^{\alpha}(i, n-i+1)} E\left(f^{\alpha-1}\left(F^{-1}\left(Z_{i}\right)\right)\right),
$$

where $Z_{i}$ has the beta distribution with parameters $\alpha(i-1)+1$ and $\alpha(n-i)+1$ and $T_{i}$ has the beta distribution with parameters $(2 \alpha-1)(i-1)+1$ and $(2 \alpha-1)(n-i)+1$.

P r o o $\mathrm{f}$ is parallel to [1, Lemma 2.1], we can prove that $\int_{s} f_{i: n}^{2 \alpha-1}(x) d x$ and $\int_{s} f_{i: n}^{\alpha}(x) d x$ are equivalent (3.2) and (3.3) respectively.

Corollary 4. The first and last Tsallis varentropy of order $\alpha$ are:

$$
\begin{aligned}
\operatorname{TVE}\left(X_{1: n}, \alpha\right) & =(1-\alpha)^{-2}\left\{\frac{n^{2 \alpha-1}}{(2 \alpha-1)(n-1)+1} E\left(f^{2 \alpha-2}\left(F^{-1}\left(T_{1}\right)\right)\right)\right. \\
& \left.-\left[\frac{n^{\alpha}}{\alpha(n-1)+1} E\left(f^{\alpha-1}\left(F^{-1}\left(Z_{1}\right)\right)\right)\right]^{2}\right\}
\end{aligned}
$$


and

$$
\begin{aligned}
\operatorname{TVE}\left(X_{n: n}, \alpha\right) & =(1-\alpha)^{-2}\left\{\frac{n^{2 \alpha-1}}{(2 \alpha-1)(n-1)+1} E\left(f^{2 \alpha-2}\left(F^{-1}\left(T_{n}\right)\right)\right)\right. \\
& \left.-\left[\frac{n^{\alpha}}{\alpha(n-1)+1} E\left(f^{\alpha-1}\left(F^{-1}\left(Z_{n}\right)\right)\right)\right]^{2}\right\} .
\end{aligned}
$$

In the following theorem we show that if $X$ has a symmetric density function with respect to $x=a$, then the Tsallis varentropy is symmetric with respect to $i$.

Theorem 7. Suppose that $X$ is a continuous random variable with the symmetric density function with respect to $x=a$, then

$$
T V E\left(X_{i: n}, \alpha\right)=T V E\left(X_{n-i+1: n}, \alpha\right) .
$$

P r o of. If $X$ has a symmetric density function with respect to $x=a$, then $X+a$ has a symmetric density with respect to $x=0$. Using the properties of order statistics $X_{i: n}+a \stackrel{d}{=}-\left(X_{n-i+1: n}+a\right)$, we have $T V E\left(X_{i: n}+a, \alpha\right)=T V E\left(-X_{n-i+1: n}-a, \alpha\right)$. Using Theorem 2, we have $T V E\left(X_{i: n}, \alpha\right)=T V E\left(X_{n-i+1: n}, \alpha\right)$.

Example 1. If $X \sim U(a, b)$ then

$$
E\left(f^{2 \alpha-2}\left(F^{-1}\left(T_{i}\right)\right)\right)=\frac{1}{(b-a)^{2 \alpha-2}} \quad \text { and } \quad E\left(f^{\alpha-1}\left(F^{-1}\left(Z_{i}\right)\right)\right)=\frac{1}{(b-a)^{\alpha-1}} .
$$

Using (3.2) and (3.3) we have:

$$
A_{i: n}(\alpha)=\frac{(b-a)^{2-2 \alpha}[B((2 \alpha-1)(i-1)+1,(2 \alpha-1)(n-i)+1)]}{B^{2 \alpha-1}(i, n-i+1)}
$$

and

$$
B_{i: n}(\alpha)=\frac{(b-a)^{1-\alpha}[B(\alpha(i-1)+1, \alpha(n-i)+1)]}{B^{\alpha}(i, n-i+1)} .
$$

Finally using (3.1) we get

$$
\begin{aligned}
\operatorname{TVE}\left(X_{i: n}, \alpha\right)= & \frac{(b-a)^{2-2 a}}{(1-\alpha)^{2}}\left\{\frac{B((2 \alpha-1)(i-1)+1,(2 \alpha-1)(n-i)+1)}{B^{2 \alpha-1}(i, n-i+1)}\right. \\
& \left.-\left[\frac{B(\alpha(i-1)+1, \alpha(n-i)+1)}{B^{\alpha}(i, n-i+1)}\right]^{2}\right\},
\end{aligned}
$$

and also

$$
\operatorname{TVE}\left(X_{1: n}, \alpha\right)=\operatorname{TVE}\left(X_{n: n}, \alpha\right)=\frac{(b-a)^{2-2 \alpha}}{(1-\alpha)^{2}}\left\{\frac{n^{2 \alpha-1}}{(2 \alpha-1)(n-1)+1}-\frac{n^{2 \alpha}}{(\alpha(n-1)+1)^{2}}\right\} .
$$

Remark 4. If $T V E\left(X_{i: n}, \alpha\right)=T V E\left(X_{n-i+1: n}, \alpha\right)$ and $T V E\left(X_{i: n}, \alpha\right)$ is decreasing with respect to $i$ for $i \leq(n+1) / 2(n / 2)$ when $n$ is odd(even), then $T V E\left(X_{i: n}, \alpha\right)$ will be increasing with respect to $i$ for $i \geq(n+1) / 2(n / 2+1)$. Therefore the median (both random variables in the middle) of order statistics has a minimum Tsallis varentropy. 


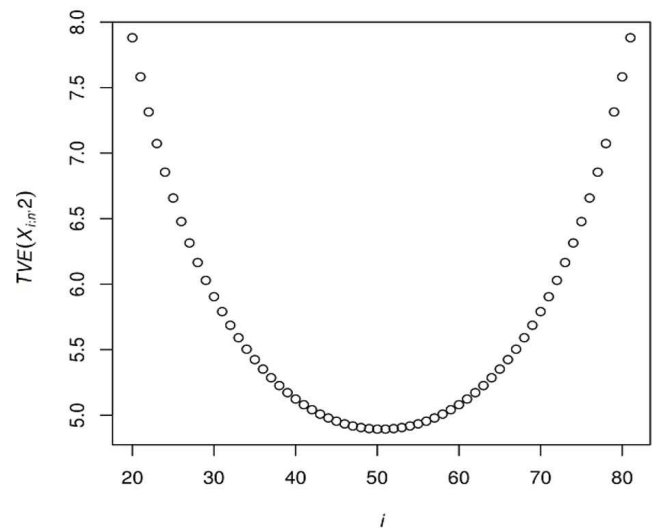

Figure 1. $T V E\left(X_{i: n}, 2\right)$ versus $i$ for the standard uniform distribution.

Figure 1 shows the Tsallis varentropy of $i$ th order statistics for the uniform distribution and it is symmetric with respect to $i$.

Example 2. If $X \sim \operatorname{Exp}(\theta)$ according to Theorem 6 we have

$$
\begin{gathered}
E\left(f^{2 \alpha-2}\left(F^{-1}\left(T_{i}\right)\right)\right)=\frac{\theta^{2 \alpha-2} B((2 \alpha-1)(i-1)+1,(2 \alpha-1)(n-i+1))}{B((2 \alpha-1)(i-1)+1,(2 \alpha-1)(n-i)+1)}, \\
E\left(f^{\alpha-1}\left(F^{-1}\left(Z_{i}\right)\right)\right)=\frac{\theta^{\alpha-1} B(\alpha(i-1)+1, \alpha(n-i+1))}{B(\alpha(i-1)+1, \alpha(n-i)+1)}
\end{gathered}
$$

and

$$
\begin{gathered}
A_{i: n}(\alpha)=\frac{\theta^{2 \alpha-2} B((2 \alpha-1)(i-1)+1,(2 \alpha-1)(n-i+1))}{B^{2 \alpha-1}(i, n-i+1)}, \\
B_{i: n}(\alpha)=\frac{\theta^{\alpha-1} B(\alpha(i-1)+1, \alpha(n-i+1))}{B^{\alpha}(i, n-i+1)}
\end{gathered}
$$

finally

$$
\begin{aligned}
T V E\left(X_{i: n}, \alpha\right)= & \frac{\theta^{2 \alpha-2}}{(1-\alpha)^{2}}\left\{\frac{B((2 \alpha-1)(i-1)+1,(2 \alpha-1)(n-i+1))}{B^{2 \alpha-1}(i, n-i+1)}\right. \\
& \left.-\left[\frac{B(\alpha(i-1)+1, \alpha(n-i+1))}{B^{\alpha}(i, n-i+1)}\right]^{2}\right\} .
\end{aligned}
$$

Figures $2 \mathrm{a}-2 \mathrm{c}$ show the Tsallis varentropy of $i$ th order statistics for the exponential distribution for $\theta=2$ and some selected values for $\alpha$. When $\alpha \rightarrow 1$, the symmetric property is observed.

\section{The Tsallis varentropy in lifetime study}

In reliability science, the hazard rate and reversed hazard rate functions are essential functions that can help engineers to analyze the system's disability. If $f$ and $\bar{F}$ are density and survival function, respectively, the hazard rate and reversed hazard functions of $X$ are $r(x)=f(x) / \bar{F}(x)$ and $\mu(x)=f(x) / F(x)$, respectively. We know that if a lifetime distribution has an increasing (decreasing) hazard rate, then it is called the $\operatorname{IFR}(D F R)$ distribution, and if it has an increasing 


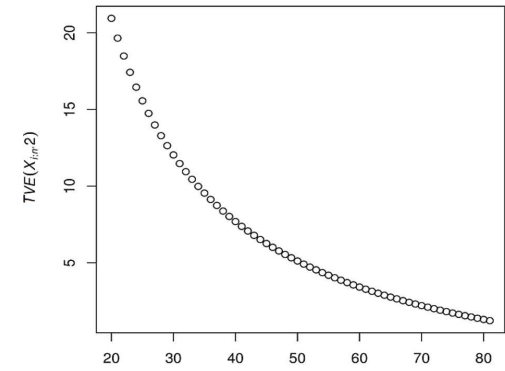

(a) $T V E\left(X_{i: n}, 2\right)$ versus $i$.

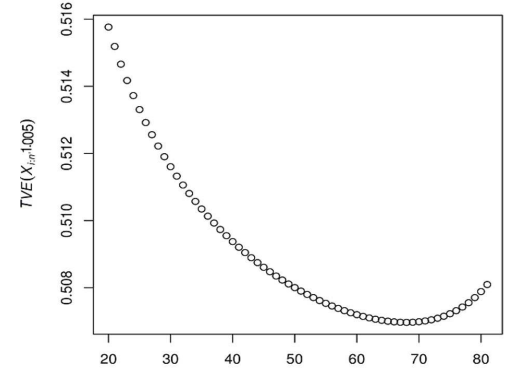

(b) $T V E\left(X_{i: n}, 1.005\right)$ versus $i$.

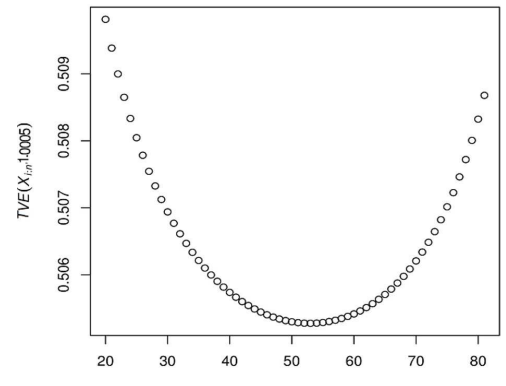

(c) $T V E\left(X_{i: n}, 1.0005\right)$ versus $i$.

Figure 2. $\operatorname{TVE}\left(X_{i: n}, \alpha\right)$ versus $i$ for the exponential distribution and $\theta=2$ and $n=100$.

(decreasing) reversed hazard rate, then it is called the $\operatorname{IRFR}(D R F R)$. In this part, we introduce some bounds by hazard and reversed hazard rate functions for $T V E$ and study them in residual (past) and double truncated lifetime distributions and also we examine the effect of system's age on them.

Theorem 8. Let $X$ be a nonnegative continuous random variable and let $r(x)$ be the hazard rate function of it. Then

(a) $\operatorname{TVE}(X, \alpha)=\frac{1}{(1-\alpha)^{2}}\left\{\operatorname{Cov}\left(r^{2 \alpha-2}(X), \bar{F}^{2 \alpha-2}(X)\right)+\frac{E\left(r^{2 \alpha-2}(X)\right)}{2 \alpha-1}-\frac{E^{2}\left(r^{\alpha-1}(X)\right)}{\alpha^{2}}\right\}$,

(b) $T V E(X, \alpha)<(>) \frac{1}{(1-\alpha)^{2}}\left\{\operatorname{Cov}\left(r^{2 \alpha-2}(X), \bar{F}^{2 \alpha-2}(X)\right)\right\}, \quad$ if $\quad 0<\alpha<\frac{1}{2} \quad\left(\alpha>\frac{1}{2}\right)$,

(c) $\operatorname{TVE}(X, \alpha)<(>) \frac{1}{(1-\alpha)^{2}}\left\{\frac{E\left(r^{2 \alpha-2}(X)\right)}{2 \alpha-1}-\frac{E^{2}\left(r^{\alpha-1}(X)\right)}{\alpha^{2}}\right\}$, if $\quad F \quad$ is $\operatorname{IFR}(D F R)$.

P r o o f. It is obvious that

$$
T V E(X, \alpha)=\frac{1}{(1-\alpha)^{2}} \operatorname{Var}\left(r^{\alpha-1}(X) \bar{F}^{\alpha-1}(X)\right) .
$$

On the other hand,

$$
\operatorname{Var}(X Y)=\operatorname{Cov}\left(X^{2}, Y^{2}\right)+E\left(X^{2}\right) E\left(Y^{2}\right)-(E(X) E(Y))^{2} .
$$

Using (4.4), we have

$$
\begin{aligned}
T V E(X, \alpha)=\frac{1}{(1-\alpha)^{2}}\{ & \operatorname{Cov}\left(r^{2 \alpha-2}(X), \bar{F}^{2 \alpha-2}(X)\right)+E\left(r^{2 \alpha-2}(X)\right) \cdot E\left(\bar{F}^{2 \alpha-2}(X)\right) \\
& \left.-\left[E\left(r^{\alpha-1}(X)\right) \cdot E\left(\bar{F}^{\alpha-1}(X)\right)\right]^{2}\right\} .
\end{aligned}
$$

Since $E\left(\bar{F}^{2 \alpha-2}(X)\right)=1 /(2 \alpha-1)$ and $E\left(\bar{F}^{\alpha-1}(X)\right)=1 / \alpha,(4.1)$ is easily obtained.

For $0<\alpha<1 / 2$, the inequality

$$
\frac{E\left(r^{2 \alpha-2}(X)\right)}{2 \alpha-1}<\frac{E^{2}\left(r^{\alpha-1}(X)\right)}{\alpha^{2}}
$$

is established and the first inequality of (4.2) is proved. 
We know that $E\left(r^{2 \alpha-2}(X)\right) \geq E^{2}\left(r^{\alpha-1}(X)\right)$ and $1 /(2 \alpha-1)>1 / \alpha^{2}$ for all $\alpha>1 / 2$. Hence

$$
\frac{E\left(r^{2 \alpha-2}(X)\right)}{2 \alpha-1}>\frac{E^{2}\left(r^{\alpha-1}(X)\right)}{\alpha^{2}}
$$

and the second inequality of (4.2) is obtained. It is easy to see that if $F$ has an $I F R$ distribution, then $r(x)$ is an increasing function of $x$, and because $\bar{F}$ is decreasing, the covariance is negative and the first inequality of (4.3) holds. The second inequality is similarly obtained.

Corollary 5. Let $X$ be a nonnegative continuous random variable and let $\mu(x)$ be the reversed hazard rate function of it, then

(a) $\operatorname{TVE}(X, \alpha)=\frac{1}{(1-\alpha)^{2}}\left\{\operatorname{Cov}\left(\mu^{2 \alpha-2}(X), F^{2 \alpha-2}(X)\right)+\frac{E\left(\mu^{2 \alpha-2}(X)\right)}{2 \alpha-1}-\frac{E^{2}\left(\mu^{\alpha-1}(X)\right)}{\alpha^{2}}\right\}$,

(b) $T V E(X, \alpha)<(>) \frac{1}{(1-\alpha)^{2}}\left\{\operatorname{Cov}\left(\mu^{2 \alpha-2}(X), F^{2 \alpha-2}(X)\right)\right\}, \quad$ if $\quad 0<\alpha<\frac{1}{2} \quad\left(\alpha>\frac{1}{2}\right)$,

(c) $\operatorname{TVE}(X, \alpha)>(<) \frac{1}{(1-\alpha)^{2}}\left\{\frac{E\left(\mu^{2 \alpha-2}(X)\right)}{2 \alpha-1}-\frac{E^{2}\left(\mu^{\alpha-1}(X)\right)}{\alpha^{2}}\right\}, \quad$ if $\quad F \quad$ is $\quad \operatorname{IRFR}(D R F R)$.

In the survival analysis and reliability engineering, we usually know the system's age. Hence (2.1) is not suitable in such a situation. The random variables $\{X-t \mid X \geq t\},\{t-X \mid X \leq t\}$ and $\left\{X \mid t_{1} \leq X \leq t_{2}\right\}$ are indicative residual, past and double truncated (interval) lifetime of the system. If $f$ and $\bar{F}$ are density function and survival function of $X$, respectively, then the residual, past and interval lifetime density functions at the time $t$ are as follows:

$$
\begin{gathered}
g_{R}(x, t)=\frac{f(x)}{\bar{F}(t)}, \quad x \geq t, \\
g_{P}(x, t)=\frac{f(x)}{F(t)}, \quad x \leq t, \\
g_{I}\left(x, t_{1}, t_{2}\right)=\frac{f(x)}{F\left(t_{2}\right)-F\left(t_{1}\right)}, \quad t_{1} \leq x \leq t_{2} .
\end{gathered}
$$

Also dynamic Tsallis entropy of $X$ for the residual, past and double truncated lifetime random variables are defined as

$$
\begin{aligned}
I_{T_{R}}(X, \alpha, t) & =\frac{1}{1-\alpha}\left[\frac{\int_{t}^{\infty} f^{\alpha}(x) d x}{\bar{F}^{\alpha}(t)}-1\right], \quad \alpha>0, \quad \alpha \neq 1, \\
I_{T_{P}}(X, \alpha, t) & =\frac{1}{1-\alpha}\left[\frac{\int_{0}^{t} f^{\alpha}(x) d x}{F^{\alpha}(t)}-1\right], \quad \alpha>0, \quad \alpha \neq 1, \\
I_{T_{I}}\left(X, \alpha, t_{1}, t_{2}\right) & =\frac{1}{1-\alpha}\left[\frac{\int_{t_{1}}^{t_{2}} f^{\alpha}(x) d x}{\left(F\left(t_{2}\right)-F\left(t_{1}\right)\right)^{\alpha}}-1\right], \quad \alpha>0, \quad \alpha \neq 1 .
\end{aligned}
$$

Definition 4. The residual, past and interval Tsallis Varentropy of nonnegative random variables $\{X-t \mid X \geq t\},\{t-X \mid X \leq t\}$ and $\left\{X \mid t_{1} \leq X \leq t_{2}\right\}$ are defined as

$$
\begin{gathered}
T V E_{R}(X, \alpha, t)=\frac{1}{(1-\alpha)^{2}} \operatorname{Var}\left(\left(\frac{f(X)}{\bar{F}(t)}\right)^{\alpha-1} \mid X \geq t\right)=\frac{\bar{F}^{2-2 \alpha}(t)}{(1-\alpha)^{2}} \operatorname{Var}\left(f^{\alpha-1}(X) \mid X \geq t\right), \\
T V E_{P}(X, \alpha, t)=\frac{1}{(1-\alpha)^{2}} \operatorname{Var}\left(\left(\frac{f(X)}{F(t)}\right)^{\alpha-1} \mid X \leq t\right)=\frac{F^{2-2 \alpha}(t)}{(1-\alpha)^{2}} \operatorname{Var}\left(f^{\alpha-1}(X) \mid X \leq t\right), \\
T V E_{I}\left(X, \alpha, t_{1}, t_{2}\right)=\frac{1}{(1-\alpha)^{2}} \operatorname{Var}\left(\left(\frac{f(X)}{F\left(t_{2}\right)-F\left(t_{1}\right)}\right)^{\alpha-1} \mid t_{1} \leq X \leq t_{2}\right) \\
=\frac{\left(F\left(t_{2}\right)-F\left(t_{1}\right)\right)^{2-2 \alpha}}{(1-\alpha)^{2}} \operatorname{Var}\left(f^{\alpha-1}(X) \mid t_{1} \leq X \leq t_{2}\right) .
\end{gathered}
$$


It is clear that when $t \rightarrow 0(t \rightarrow \infty), T V E_{R}(X, \alpha, t)\left(T V E_{P}(X, \alpha, t)\right)=T V E(X, \alpha)$ and if $t_{1} \rightarrow 0$, $t_{2} \rightarrow \infty$, then $T V E_{I}\left(X, t_{1}, t_{2}\right)=T V E(X, \alpha)$. For example, if $X$ has a Pareto distribution with density function

$$
f(x)=\frac{\theta \beta^{\theta}}{x^{\theta+1}}, \quad x>\beta, \quad \beta>0, \quad \theta>0, \quad \bar{F}(t)=\frac{\beta^{\theta}}{t^{\theta}},
$$

then

$$
\begin{gathered}
T V E_{R}(X, \alpha, t)=\frac{t^{\theta(2 \alpha-2)} \theta^{2 \alpha-2}}{(1-\alpha)^{2}} \operatorname{Var}\left(X^{(\theta+1)(1-\alpha)} \mid X \geq t\right), \\
T V E_{R}(X, \alpha, t)=\frac{t^{2-2 \alpha} \theta^{2 \alpha}}{(1-\alpha)^{2}}\left\{\frac{-1}{\theta(\theta+1)(1-2 \alpha)+\theta}+\frac{1}{[-\alpha(\theta+1)+1]^{2}}\right\} .
\end{gathered}
$$

If $\alpha \rightarrow 1$, then the Tsallis residual varentropy reduces to the residual varentropy of Pareto distribution. It is $(\theta+1)^{2} / \theta^{2}$ for all $t>0$, and that is independent of the age of systems, but the Tsallis residual varentropy is not.

Theorem 9. $X$ has a uniform distribution if and only if $T V E_{R}(X, \alpha, t)=0, T V E_{P}(X, \alpha, t)=$ 0 , or $T V E_{I}\left(X, t_{1}, t_{2}\right)=0$.

P r o o f. If $X \sim U(a, b)$, then

$$
T V E_{R}(X, \alpha, t)=\frac{\bar{F}^{2-2 \alpha}(t)}{(1-\alpha)^{2}} \operatorname{Var}\left((b-a)^{1-\alpha} \mid X \geq t\right)=0 .
$$

On the other hand if $T V E_{R}(X, \alpha, t)=0$, then

$$
\frac{\bar{F}^{2-2 \alpha}(t)}{(1-\alpha)^{2}} \operatorname{Var}\left(f(X)^{\alpha-1} \mid X \geq t\right)=0
$$

and $f(X)$ is almost surely constant. Similar to Theorem $1, X$ has the uniform distribution. For the other two cases, the proof is the same.

Proposition 2. If $X$ has an exponential distribution, then the Tsallis residual varentropy is independent of lifetime of systems.

P r o o f. In the exponential case, we know

$$
g_{R}(x, t)=\frac{f(x+t)}{\bar{F}(t)}=\theta e^{-\theta x}, \quad x>0 .
$$

Therefore the residual lifetime distribution is independent of $t$ and $g_{R}(x, t)=f(x)$ and $T V E_{R}(X, \alpha, t)=T V E(X, \alpha)$.

We can introduce two new classes of distributions using the following definition.

Definition 5. We say that $\bar{F}$ has an increasing (decreasing) Tsallis residual varentropy ITRVE $(D T R V E)$ if $T V E_{R}(X, \alpha, t)$ is an increasing (decreasing) function of $t$, and $F$ has an increasing (decreasing) Tsallis past varentropy ITPVE(DTPVE) if TVE $E_{P}(X, \alpha, t)$ is an increasing (decreasing) function of $t$ for all $t \geq 0$.

Theorem 10. $\bar{F}(F)$ has DTRVE(ITPVE) in $t \geq 0$ if $T V E_{R}(X, \alpha, t)\left(T V E_{P}(X, \alpha, t)\right)<\infty$, $I_{T_{R}}(X, \alpha, t)\left(I_{T_{P}}(X, \alpha, t)\right)<\infty$, and $0<\alpha \leq 1 / 2$. 
P r o o f. Using the differentiation of (4.5) and (4.6) with respect to $t$, we have

$$
\begin{gathered}
(1-\alpha)^{2} T V E_{R}^{\prime}(X, \alpha, t)=r(t)\left\{(2 \alpha-1)(1-\alpha)^{2} T V E_{R}(X, \alpha, t)\right. \\
\left.-\left[(1-\alpha) I_{T_{R}}(X, \alpha, t)+1-r^{\alpha-1}(t)\right]^{2}\right\}, \\
(1-\alpha)^{2} T V E_{P}^{\prime}(X, \alpha, t)=\mu(t)\left\{(1-2 \alpha)(1-\alpha)^{2} T V E_{P}(X, \alpha, t)\right. \\
\left.+\left[\mu^{\alpha-1}(t)-(1-\alpha) I_{T_{P}}(X, \alpha, t)-1\right]^{2}\right\},
\end{gathered}
$$

where $I_{T_{R}}(X, \alpha, t)$ and $I_{T_{P}}(X, \alpha, t)$ are the Tsallis residual and past entropy of $X$ respectively. We see that if $0<\alpha \leq 1 / 2$ then $T V E_{R}^{\prime}(X, \alpha, t)\left(T V E_{P}^{\prime}(X, \alpha, t)\right) \leq(\geq) 0$ and $\bar{F}(F)$ has $D T R V E(I T P V E)$.

Theorem 11. $\bar{F}$ has ITRVE(DTRVE) in $t \geq 0$ if $T V E_{R}(X, \alpha, t)<\infty, I_{T_{R}}(X, \alpha, t)<\infty$, and for all $\alpha>1 / 2$,

$$
(2 \alpha-1)(1-\alpha)^{2} T V E_{R}(X, \alpha, t) \geq(\leq)\left[(1-\alpha) I_{T_{R}}(X, \alpha, t)+1-r^{\alpha-1}(t)\right]^{2} .
$$

Also $F$ has DTPVE(ITPVE) in $t \geq 0$ if $T V E_{P}(X, \alpha, t)<\infty, I_{T_{P}}(X, \alpha, t)<\infty$, and for all $\alpha>1 / 2$,

$$
|1-2 \alpha|(1-\alpha)^{2} T V E_{P}(X, \alpha, t) \geq(\leq)\left[\mu^{\alpha-1}(t)-(1-\alpha) I_{T_{P}}(X, \alpha, t)-1\right]^{2} .
$$

P r o of. In Definition $5 \bar{F}$ has $\operatorname{ITRVE}(D T R V E)$ in $t$ if $T V E_{R}^{\prime}(X, \alpha, t) \geq(\leq) 0$. By using (4.8), the proof is completed. Also (4.10) can be similarly proved by using (4.9).

Corollary 6. If $\bar{F}$ has ITRVE $(D T R V E)$ in $t \geq 0$, then for all $\alpha>1 / 2$

$$
\operatorname{TVE}(X, \alpha) \geq(\leq) \frac{\left[(1-\alpha) I_{T}(X, \alpha)+1-f^{\alpha-1}(0)\right]^{2}}{(2 \alpha-1)(1-\alpha)^{2}} .
$$

And if $F$ has DTPVE(ITPVE) in $t \geq 0$, then for all $\alpha>1 / 2$

$$
\operatorname{TVE}(X, \alpha) \geq(\leq) \frac{\left[f^{\alpha-1}(\infty)-(1-\alpha) I_{T}(X, \alpha)-1\right]^{2}}{|1-2 \alpha|(1-\alpha)^{2}} .
$$

Therefore (4.11) and (4.12) are lower (upper) bound for Tsallis varentropy for all $\alpha>1 / 2$.

Corollary 7. Let $\bar{F}$ be both ITRVE $(D T R V E)$, so $T V E_{R}^{\prime}(X, \alpha, t)=0$. Then

$$
(2 \alpha-1)(1-\alpha)^{2} T V E_{R}(X, \alpha, t)=\left[(1-\alpha) I_{T_{R}}(X, \alpha, t)+1-r^{\alpha-1}(t)\right]^{2}, \quad \alpha>1 / 2,
$$

and

$$
T V E(X, \alpha)=\frac{\left[(1-\alpha) I_{T}(X, \alpha)+1-f^{\alpha-1}(0)\right]^{2}}{(2 \alpha-1)(1-\alpha)^{2}}, \quad \alpha>\frac{1}{2}
$$

and if $F$ is both ITPVE $(D T P V E)$, then $T V E_{P}^{\prime}(X, \alpha, t)=0$ and we have

$$
|1-2 \alpha|(1-\alpha)^{2} T V E_{P}(X, \alpha, t)=\left[\mu^{\alpha-1}(t)-(1-\alpha) I_{T_{P}}(X, \alpha, t)-1\right]^{2},
$$

therefore

$$
T V E(X, \alpha)=\frac{\left[f^{\alpha-1}(\infty)-(1-\alpha) I_{T}(X, \alpha)-1\right]^{2}}{|1-2 \alpha|(1-\alpha)^{2}}, \quad \alpha>\frac{1}{2}
$$

Therefore (4.13) and (4.14) introduce the Tsallis varentropy when system's age is ineffective on it. 


\section{Conclusion}

In this paper, we introduced the generalized varentropy of order $\alpha$ for continuous random variables based on the Tsallis entropy. We showed that unlike the varentropy, which is a location and scale-invariant measure, the Tsallis varentropy is invariant to the location transformation but is not invariant to scale translate, unless when $\alpha \rightarrow 1$. After presenting some theorems of the properties of the Tsallis varentropy, we investigated them in the order statistics, which can be useful for the system designers in the lifetime information for the $(n-i+1)$-out-of- $n$ systems. Also we studied them for the lifetime distributions and obtained some bounds for them by using the hazard and reversed hazard rate functions. Then we studied the age of systems regarding residual lifetime distributions and showed that in the uniform and exponential distributions, Tsallis residual varentropy is independent of the age of systems. We introduced two new classes of distributions by using the residual and past Tsallis varentropy, and we described some its properties.

\section{Acknowledgements}

The authors would like to thank the editor and anonymous referees for their valuable comments and suggestions that improved the quality of the paper.

\section{REFERENCES}

1. Abbasnejad M., Arghami N.R. Renyi entropy properties of order statistics. Comm. Statist. Theory Methods, 2010. Vol. 40, No. 1. P. 40-52. DOI: 10.1080/03610920903353683

2. Afhami B., Madadi M., Rezapour M. Goodness-of-fit test based on Shannon entropy of $k$-record values from the generalized. J. Stat. Sci., 2015. Vol. 9, No. 1. P. 43-60.

3. Arikan E. Varentropy decreases under the polar transform. IEEE Trans. Inform. Theory, 2016. Vol. 62, No. 6. P. 3390-3400. DOI: 10.1109/TIT.2016.2555841

4. Arnold B. C., Balakrishnan N., Nagaraja H. N. A First Course in Order Statistics. Classics Appl. Math., vol. 54. Philadelphia: SIAM, 2008. 279 p. DOI: 10.1137/1.9780898719062

5. Baratpour S., Ahmadi J., Arghami N.R. Characterizations based on Rényi entropy of order statistics and record values. J. Statist. Plann. Inference, 2008. Vol. 138, No. 8. P. 2544-2551. DOI: $10.1016 /$ j.jspi.2007.10.024

6. Baratpour S., Khammar A. Tsallis entropy properties of order statistics and some stochastic comparisons. J. Statist. Res. Iran, 2016. Vol. 13, No. 1. P. 25-41. DOI: 10.18869/acadpub.jsri.13.1.2

7. Bobkov S., Madiman M. Concentration of the information in data with log-concave distributions. Ann. Probab., 2011. Vol. 39, No. 4. P. 1528-1543. URL: https://projecteuclid.org/euclid.aop/1312555807

8. David H. A., Nagaraja H. N. Order Statistics. 3rd edition. Wiley Ser. Probab. Stat. Hoboken, New Jersey: John Wiley \& Sons, Inc. 2003. 458 p. DOI: 10.1002/0471722162

9. Di Crescenzo A., Longobardi M. Statistic comparisons of cumulative entropies. In: Stochastic Orders in Reliability and Risk. Li H., Li X. (eds.). Lect. Notes Stat., vol. 208. New York: Springer, 2013. P. 167-182. DOI: 10.1007/978-1-4614-6892-9_8

10. Di Crescenzo A., Paolillo L. Analysis and applications of the residual varentropy of random lifetimes. Probab. Engrg. Inform. Sci., 2020. P. 1-19. DOI: 10.1017/S0269964820000133

11. Ebrahimi N., Kirmani S. N. U.A. Some results on ordering of survival functions through uncertainty. Statist. Probab. Lett., 1996. Vol. 29, No. 2. P. 167-176. DOI: 10.1016/0167-7152(95)00170-0

12. Ebrahimi N., Soofi E. S., Zahedi H. Information properties of order statistics and spacing. IEEE Trans. Inform. Theory, 2004. Vol. 50, No. 1. P. 177-183. DOI: 10.1109/TIT.2003.821973

13. Enomoto R., Okamoto N., Seo T. On the asymptotic normality of test statistics using Song's kurtosis. J. Stat. Theory Pract., 2013. Vol. 7, No. 1. P. 102-119. DOI: 10.1080/15598608.2013.756351

14. Gupta R. C., Taneja H.C., Thapliyal R. Stochastic comparisons based on residual entropy of order statistics and some characterization results. J. Stat. Theory Appl., 2014. Vol. 13, No. 1. P. 27-37. DOI: $10.2991 /$ jsta.2014.13.1.3 
15. Kontoyiannis I., Verdú S. Optimal lossless compression: Source varentropy and dispersion. IEEE Trans. Inform. Theory, 2014. Vol. 60, No. 2. P. 777-795. DOI: 10.1109/TIT.2013.2291007

16. Liu J. Information Theoretic Content and Probability. Ph.D. Thesis, University of Florida, 2007.

17. Nanda A. K., Paul P. Some results on generalized residual entropy. Inform. Sci., 2006. Vol. 176, No. 1. P. 27-47. DOI: 10.1016/j.ins.2004.10.008

18. Park S. The entropy of consecutive order statistics. IEEE Trans. Inform. Theory, 1995. Vol. 41, No. 6. P. 2003-2007. DOI: 10.1109/18.476325

19. Psarrakos G., Navarro J. Generalized cumulative residual entropy and record values. Metrika, 2013. Vol. 76. P. 623-640. DOI: 10.1007/s00184-012-0408-6

20. Raqab M.Z., Amin W. A. Some ordering result on order statistics and record values. IAPQR Trans., 1996. Vol. 21, No. 1. P. 1-8.

21. Shannon C.E. A mathematical theory of communication. Bell System Technical J., 1948. Vol. 27, No. 3. P. 379-423 DOI: 10.1002/j.1538-7305.1948.tb01338.x

22. Song K.-S. Rényi information, log likelihood and an intrinsic distribution measure. J. Statist. Plann. Inference, 2001. Vol. 93, No. 1-2. P. 51-69. DOI: 10.1016/S0378-3758(00)00169-5

23. Tsallis C. Possible generalization of Boltzmann-Gibbs statistics. J. Stat. Phys., 1988. Vol. 52. P. $479-487$. DOI: 10.1007/BF01016429

24. Vikas Kumar, Taneja H. C. A generalized entropy-based residual lifetime distributions. Int. J. Biomath., 2011. Vol. 04, No. 02. P. 171-148. DOI: 10.1142/S1793524511001416

25. Wilk G., Włodarczyk Z. Example of a possible interpretation of Tsallis entropy. Phys. A: Stat. Mech. Appl., 2008. Vol. 387, No. 19-20. P. 4809-4813. DOI: 10.1016/j.physa.2008.04.022

26. Wong K. M., Chen S. The entropy of ordered sequences and order statistics. IEEE Trans. Inform. Theory, 1990. Vol. 36, No. 2. P. 276-284. DOI: 10.1109/18.52473

27. Zarezadeh S., Asadi M. Results on residual Rényi entropy of order statistics and record values. Inform. Sci., 2010. Vol. 180, No. 21. P. 4195-4206. DOI: 10.1016/j.ins.2010.06.019

28. Zhang Z. Uniform estimates on the Tsallis entropies. Lett. Math. Phys., 2007. Vol. 80. P. $171-181$. DOI: $10.1007 / \mathrm{s} 11005-007-0155-1$

29. Zografos K. On Mardia's and Song's measures of kurtosis in elliptical distributions. J. Multivariate Anal., 2008. Vol. 99, No. 5. P. 858-879. DOI: 10.1016/j.jmva.2007.05.001 